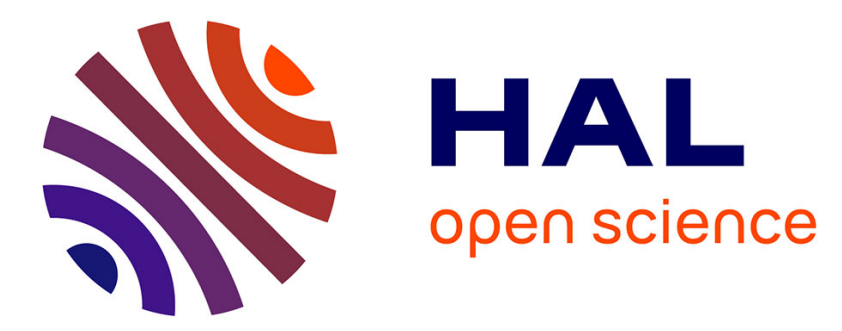

\title{
Vers une plus grande flexibilité temporelle du modèle opérationnel de prévision des crues GRP
}

J. Viatgé, Thomas Pinna, A. Ficchì, Charles Perrin, D. Dorchies, L. Garandeau, F. Tilmant

\section{- To cite this version:}

J. Viatgé, Thomas Pinna, A. Ficchì, Charles Perrin, D. Dorchies, et al.. Vers une plus grande flexibilité temporelle du modèle opérationnel de prévision des crues GRP. La Houille Blanche - Revue internationale de l'eau, 2019, 2, pp.72-80. 10.1051/lhb/2019017 . hal-02609669

\section{HAL Id: hal-02609669 \\ https: / hal.inrae.fr/hal-02609669}

Submitted on 16 May 2020

HAL is a multi-disciplinary open access archive for the deposit and dissemination of scientific research documents, whether they are published or not. The documents may come from teaching and research institutions in France or abroad, or from public or private research centers.
L'archive ouverte pluridisciplinaire HAL, est destinée au dépôt et à la diffusion de documents scientifiques de niveau recherche, publiés ou non, émanant des établissements d'enseignement et de recherche français ou étrangers, des laboratoires publics ou privés. 


\title{
Vers une plus grande flexibilité temporelle du modèle opérationnel de prévision des crues GRP
}

\author{
Julie Viatgé ${ }^{1,{ }^{*}}$, Thomas Pinna ${ }^{1, \mathrm{a}}$, Andrea Ficchì ${ }^{1, \mathrm{~b}}$, Charles Perrin ${ }^{1}$, David Dorchies ${ }^{2}$, Léa Garandeau ${ }^{3}$ \\ et François Tilmant ${ }^{1}$ \\ ${ }^{1}$ Irstea, UR HYCAR, 1 rue Pierre-Gilles de Gennes, CS 10030, 92761 Antony Cedex, France \\ 2 Irstea, UMR G-Eau, 361 rue J.F. Breton, BP 5095, 34196 Montpellier Cedex 5, France \\ ${ }^{3}$ SCHAPI, 42, avenue Gaspard Coriolis, 31057 Toulouse, France
}

Reçu le 16 février 2018 / Accepté le 26 septembre 2018

\begin{abstract}
Résumé - La prévision des crues est souvent réalisée au pas de temps horaire, qui est un bon compromis entre disponibilité des données et résolution nécessaire pour bien rendre compte des phénomènes de crue. Cependant, dans un certain nombre de cas, ce pas de temps fixe est limitant, et des pas de temps plus fins (ou éventuellement plus larges) sont mieux adaptés. Il est donc nécessaire d'avoir des modèles hydrologiques capables de s'adapter à un fonctionnement à différents pas de temps. Or, cette adaptation ne se résume généralement pas à un simple changement de la longueur du pas de temps dans les équations du modèle. Elle implique une explicitation de la dépendance possible de certains paramètres du modèle au pas de temps, et également une adaptation de la structure du modèle pour que les processus soient représentés de manière cohérente entre les pas de temps. L'article présente une adaptation du modèle hydrologique GRP, qui est largement utilisé en conditions opérationnelles par les services de prévision des crues français, pour qu'il fonctionne correctement à différents pas de temps. Les tests sont réalisés sur une base de 240 bassins versants français.
\end{abstract}

Mots clés : prévision des crues / modélisation hydrologique / pas de temps / modèle GRP

\begin{abstract}
Towards an enhanced temporal flexibility of the GRP flood forecasting operational model. Nowadays, flood forecasting is often done at the hourly time step, which is a good compromise between data availability and the necessary resolution to simulate flood events. However, in a number of cases, this fixed time step shows limitations, and shorter (or even larger) time steps are required. Therefore, it is necessary to have hydrological models able to adapt to various time steps. Generally, this adaptation does not limit to a simple change of the length of the time step in the model equations. The time-step dependency must be made explicit in some model parameters, and the model structure must also be adapted to consistently represent processes across time steps. The article presents the adaptation of the GRP hydrological model, which is widely used by flood forecasting services in operational conditions in France, so that it can behave and run consistently at different time steps. The tests were made on a set of 240 French catchments.
\end{abstract}

Keywords: flood forecasting / hydrological modelling / time step / GRP model

\footnotetext{
*Correspondance : julie.viatge@irstea.fr

${ }^{a}$ Adresse personnelle: Sorbonne Université UMR METIS, 4 place Jussieu, 75252 Paris Cedex 05, France.

b Adresse personnelle: University of Reading, Geography and Environmental Science, Whiteknights, PO Box 217, Reading, Berkshire, RG6 6AH, Royaume-Uni.
} 


\section{Introduction}

\subsection{Contexte}

L'outil de prévision des crues GRP, actuellement utilisé par une grande partie des services de prévisions des crues (SPC) français (voir son utilisation en 2018 à la Fig. 1), est fondé sur un modèle hydrologique qui fonctionne en continu au pas de temps horaire. Il permet d'estimer les débits futurs sur des cours d'eau jaugés à partir d'observations et de prévisions de pluie. Ce modèle a été développé par Irstea en collaboration avec le réseau Vigicrues (Service central d'hydrométéorologie et d'appui à la prévision des inondations - SCHAPI - et SPC) et continue d'évoluer grâce, notamment, aux retours d'expérience des prévisionnistes du réseau. Des rencontres entre les utilisateurs et l'équipe de développement d'Irstea sont organisées chaque année dans l'optique de mieux cerner les limites du modèle et de proposer des axes de développement pour répondre à ces difficultés (Furusho et al., 2015, 2016).

$\mathrm{Au}$ cours des dernières années, le pas de temps fixe, horaire, du modèle est apparu être un facteur limitant pour son utilisation dans certains cas. En effet, le pas de temps horaire convient bien à des bassins versants ayant des temps de réaction supérieurs à quelques heures, mais devient trop grossier pour des bassins versants plus réactifs. D'autre part, le modèle ne peut exploiter toute l'information contenue dans des données à des pas de temps de quelques minutes (par ex. issues des radars) qui sont aujourd'hui largement disponibles, ni inversement, s'adapter efficacement à la disponibilité seulement journalière ou pluri-horaire d'autres données (par ex. prévisions symposium «RR3» sur trois heures).

Cela a donc conduit à travailler au développement d'un modèle GRP pouvant fonctionner à des pas de temps multiples, couvrant une gamme allant de l'infra-horaire au journalier.

\subsection{Enjeu de l'adaptation à des pas de temps multiples}

La modélisation hydrologique opère une triple simplification de la transformation pluie-débit, en termes de processus représentés, d'échelle spatiale et d'échelle temporelle choisies. Si les deux premiers aspects ont été assez largement étudiés dans la littérature scientifique, la question du choix et de l'influence de la résolution temporelle reste assez peu explorée. Souvent négligée, cette dimension temporelle demeure cependant source d'erreurs en modélisation. Les travaux récents de Ficchì (2017) sur le modèle GR4J (Perrin et al., 2003) ont notamment montré qu'une même structure hydrologique, appliquée de manière indifférenciée sur une large gamme de pas de temps (de l'infra-horaire jusqu'au journalier) donne des résultats non cohérents, avec en particulier des erreurs sur les volumes d'eau écoulés s'amplifiant à mesure que le pas de temps diminue (Ficchì et al., 2016). Les sources de ces incohérences peuvent être multiples: dépendance des paramètres au pas de temps, procédure d'intégration numérique temporelle, ou encore prise en compte des processus en jeu. Une analyse détaillée des résultats, par évaluation de la cohérence des flux au sein du modèle à différents pas de temps, a permis de montrer que la mauvaise prise en compte du processus d'interception, dont le temps caractéristique est journalier, à des pas de temps

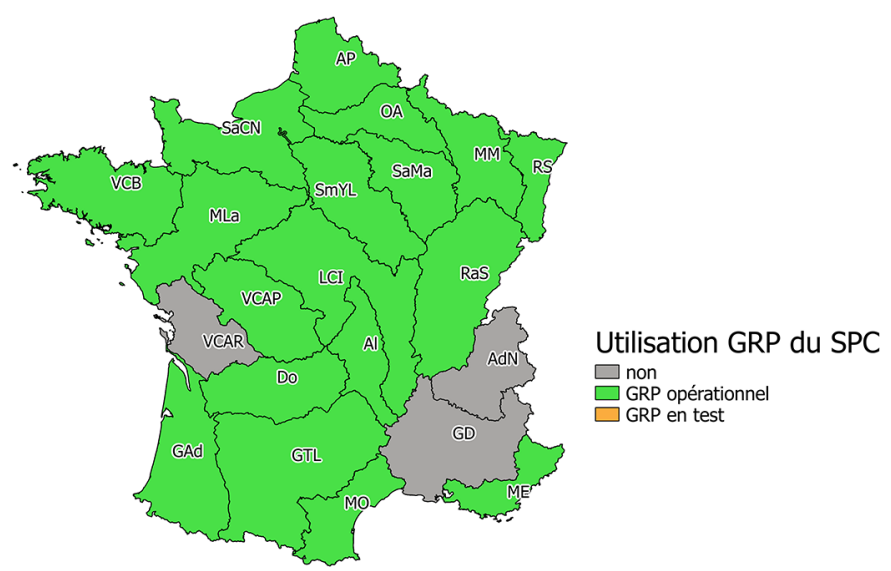

Fig. 1. Carte d'utilisation du modèle GRP au sein des Services de Prévision des Crues (SPC) français métropolitains en 2018. En vert, les SPC utilisant le modèle GRP en opérationnel; en orange les SPC dans lesquels le modèle GRP est en cours de test; en gris les SPC n'utilisant pas le modèle GRP. Les lettres correspondent aux initiales des SPC (Source: SCHAPI; voir http://www.vigicrues.gouv.fr pour le détail des noms des SPC).

inférieurs à la journée, conduit à sous-estimer les flux d'eau interceptés, et à générer des phénomènes de compensation au sein de la structure du modèle, qui limitent ses performances (Ficchì et al., 2019).

Les questions de représentation des processus dominants, des échelles d'espace et de temps sont en fait intrinsèquement liées. Plusieurs travaux ont montré par exemple la dépendance de la structure du modèle au pas de temps (voir par ex. Mouelhi, 2003) ou encore la dépendance entre pas d'espace et pas de temps (voir par ex. Obled et al., 2009; Goullet, 2016). Ici, nous nous limiterons cependant à la seule dimension temporelle.

\subsection{Objectif des travaux}

Les travaux de Ficchì (2017) ont montré l'importance du pas de temps et la nécessité d'adapter la structure du modèle GR à des pas de temps infra-horaires, en particulier en tenant mieux compte du phénomène d'interception. Ces travaux, réalisés en simulation, ouvrent la voie à une potentielle amélioration du modèle GRP en prévision. Des analyses exploratoires ont été réalisées sur ce modèle dans le cadre des travaux de Pinna (2017). L'objectif de cet article est de présenter les principaux enseignements de ces tests, et les perspectives ouvertes. Nous ne présentons cependant ici que les résultats de la version au pas de temps horaire, en privilégiant l'analyse de différentes options de modélisation.

\section{D'un modèle GRP horaire à un modèle multi-pas de temps}

\subsection{Le modèle GRP initial}

Le modèle GRP actuellement exploité dans l'outil utilisé en conditions opérationnelles (Furusho et al., 2017) a été développé par Berthet (2010), qui avait adapté au pas de temps horaire la structure initialement proposée par Tangara 
(2005) au pas de temps journalier. Il s'agit d'un modèle hydrologique global à réservoirs (voir Fig. 2), apparenté aux modèles conceptuels, fonctionnant en continu au pas de temps horaire. Il reçoit comme entrée les précipitations et l'évapotranspiration potentielle (ETP) à l'échelle du bassin versant. La structure comporte un module de production et un module de transfert.

Le module de production comporte:

- une fonction de neutralisation qui modélise l'interception des précipitations et l'évaporation correspondante au taux potentiel via un réservoir de capacité nulle;

- un réservoir de production qui permet de déterminer le rendement des pluies et de l'évapotranspiration. Ce réservoir se vidange en une percolation (Perc) qui s'ajoute à la pluie résiduelle $(P n-P s)$ pour fournir la pluie efficace $(\operatorname{Pr})$;

- une fonction de correction des pluies efficaces.

Le module de routage ne simule qu'une composante d'écoulement et comporte :

- une répartition temporelle de la pluie efficace par un hydrogramme unitaire symétrique, qui permet notamment de simuler le temps de montée au pic;

- un réservoir de routage qui permet de simuler la dynamique de crue et la récession.

En prévision, le modèle est utilisé conjointement avec une procédure d'assimilation de données, qui permet d'exploiter les débits observés en temps réel, en deux étapes:

- la mise à jour du réservoir de routage à partir de l'information de dernier débit observé, de sorte que le débit produit par le réservoir de routage soit égal au débit observé à l'instant de prévision;

- l'exploitation de l'erreur antérieure pour corriger les prévisions futures. Elle peut être réalisée via deux méthodes: la méthode autorégressive dite de Tangara (2005), qui calcule le ratio entre le débit observé et le débit prévu à un pas de temps lors de la dernière prévision, et l'élève à la puissance 0,45 ; et les réseaux de neurones artificiels (Berthet, 2010), qui prennent en entrée : le débit observé à l'instant $t$ et les erreurs additives aux pas de temps $t-1$ et $t$, où $t$ est l'instant de prévision.

Le modèle GRP possède trois paramètres à caler que sont :

- le facteur d'ajustement de la pluie efficace CORR (-) qui permet de rendre compte d'autres composantes du bilan en eau (par ex. d'éventuels échanges souterrains);

- le temps de base de l'hydrogramme unitaire TB (h) qui permet de tenir compte du décalage temporel entre pluie et débit ;

- la capacité du réservoir de routage ROUT (mm) qui permet de réaliser un lissage temporel des pluies efficaces. ROUT représente le volume maximal stockable dans le réservoir après sa vidange, et peut donc être interprétée comme une capacité à un pas de temps.

Le réservoir de production a une capacité (CAP) fixée empiriquement à $275 \mathrm{~mm}$ (Berthet, 2010) et le réservoir d'interception a une capacité nulle. GRP a également d'autres paramètres fixes, comme le coefficient de percolation.

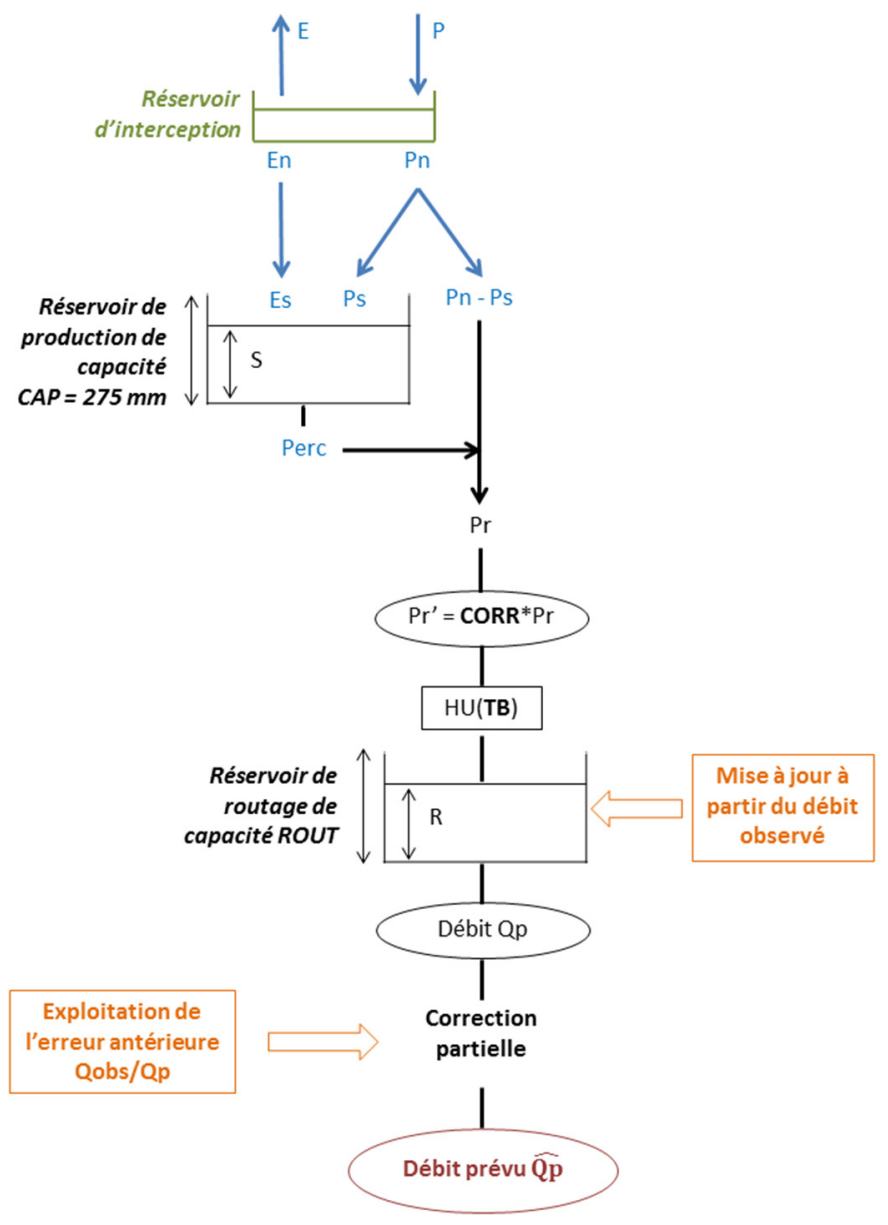

Fig. 2. Schéma de la structure du modèle GRP adapté au fonctionnement multi-pas de temps. Dans la version initiale du modèle GRP, la capacité du réservoir d'interception est nulle.

\subsection{Le modèle GRP multi-pas de temps}

\subsubsection{Ajout d'un réservoir d'interception de capacité fixe}

Les travaux de thèse de Ficchì (2017) ont permis de montrer que l'utilisation de la fonction de neutralisation pour représenter l'interception n'était pas valable à des pas de temps infra-journaliers. En effet, par ses travaux sur le modèle GR4, Ficchì (2017) a constaté une incohérence des flux interceptés entre les pas de temps. L'interception étant un processus ayant une dynamique typiquement journalière, un réservoir de capacité nulle convient bien à ce pas de temps (l'ETP journalière agissant directement sur les pluies journalières). Cependant, à pas de temps plus fin, il est nécessaire d'avoir un stockage des pluies, de manière à ce que la reprise évaporatoire puisse être différée, en fonction de l'énergie disponible sur la journée. Un réservoir de capacité non nulle est donc nécessaire. Dans ses travaux, Ficchì a proposé un réservoir de capacité fixe (c'est-à-dire non optimisée par calage), de manière à ne pas rajouter de complexité paramétrique. La capacité du réservoir est fixée à une valeur permettant de retrouver la même quantité d'eau interceptée qu'au pas de temps journalier. Cette capacité est simplement recherchée par essai-erreur en se basant sur les deux seules entrées climatiques du modèle (pluie et ETP). Sur un échantillon de 240 bassins versants français, la capacité 
médiane identifiée au pas de temps horaire est de l'ordre de $2 \mathrm{~mm}$. De manière intéressante, Ficchì a montré que cette modification du modèle permettait d'éviter des phénomènes de compensation avec d'autres fonctions du modèle qui étaient alors nécessaires pour équilibrer le bilan en eau. Cette modification a donc été testée dans le modèle GRP.

\subsubsection{Adaptation des paramètres}

Certains paramètres des modèles hydrologiques dépendent théoriquement du temps. Il s'agit par exemple de constantes de vidange ou capacité de réservoirs de transfert, ou de temps de base d'hydrogrammes unitaires. Les travaux réalisés par exemple sur le passage du modèle GR4J à un pas de temps plus fin, ont permis d'expliciter cette dépendance (Mathevet, 2005 ; Le Moine, 2008) et donc de rendre les paramètres du modèle davantage transposables entre des pas de temps différents. Cela ouvre potentiellement la possibilité d'avoir des paramètres du modèle devenant des valeurs intrinsèques indépendantes du pas de temps. Ainsi, les paramètres du modèle déterminés au pas de temps horaire deviendraient utilisables pour d'autres pas de temps de fonctionnement.

Au sein du modèle GRP, trois paramètres dépendent du pas de temps : le temps de base TB de l'hydrogramme unitaire, la capacité ROUT du réservoir de routage et le paramètre (fixe) de la fonction de percolation. L'annexe 1 donne une formulation du modèle GRP et de ses paramètres, explicitant la dépendance au pas de temps.

\subsubsection{Autres modifications}

Les retours d'expérience reçus ces dernières années ont pointé, dans certaines conditions, des limites du modèle GRP, qui semblent liées à plusieurs aspects, notamment :

- le fait d'avoir certains paramètres fixes, par exemple la capacité du réservoir de production, introduit des contraintes fortes dans le fonctionnement de l'outil. En particulier, le modèle ne dispose que d'un seul paramètre (CORR) pour ajuster son bilan, ce qui est parfois insuffisant. Il peut alors être intéressant de libérer certains de ces paramètres lors du calage ;

- le paramètre CORR abat de manière indifférenciée, notamment indépendamment de la saison ou des intensités considérées, toutes les pluies efficaces, ce qui peut conduire à des approximations dans le fonctionnement réel du bassin versant et une difficulté d'estimation des volumes écoulés en crue. Par ailleurs, la fonction multiplicative ne permet pas de borner les échanges en valeur absolue, ce qui conduit à des pertes en eau parfois peu réalistes. Il est alors intéressant de voir si CORR peut être avantageusement remplacé par des fonctions plus complexes, introduisant par exemple la dépendance de la correction à un indice saisonnier ou événementiel ;

- l'optimisation des paramètres du modèle, qui est faite avec une fonction objective tenant compte de l'erreur quadratique à un horizon donné, peut introduire une instabilité du paramétrage entre échéances cibles, indiquant potentiellement un manque d'information lors du calage pour estimer de manière robuste ces paramètres. Cela peut venir du fait que l'assimilation de données masque une partie de l'information sur la dynamique réelle du bassin. Il peut alors être intéressant de combiner le calage de certains paramètres en prévision (avec assimilation à échéance

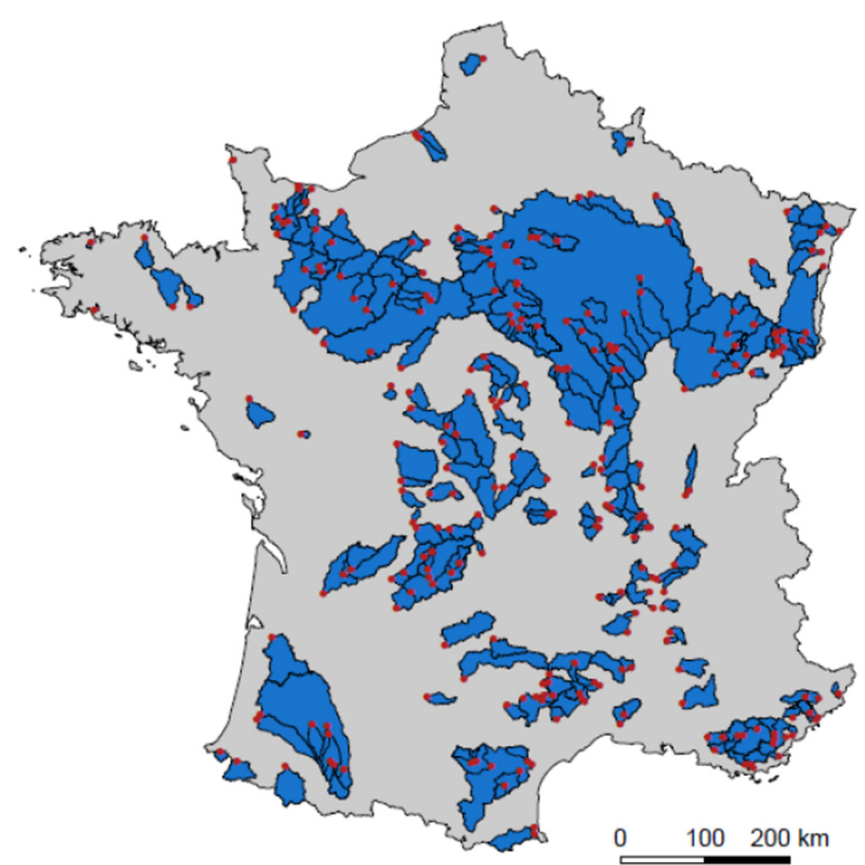

Fig. 3. Carte de 266 bassins versants de l'étude (en bleu) et leurs exutoires (en rouge).

donnée) avec le calage d'autres paramètres en simple simulation (sans assimilation). Un calage séquentiel, associant un calage d'abord en mode simulation de tous les paramètres, puis un calage en mode prévision de certains paramètres seulement (les autres étant laissés à leur valeur précédemment obtenue) a été testé.

\section{Bassins et données}

Afin de tester la transposabilité temporelle du modèle GRP, nous nous sommes appuyés sur la base de 240 bassins versants constituée par Ficchì (2017), et complétée par Pinna (2017) avec 26 bassins supplémentaires situés sur les zones de compétence des SPC Loire-Cher-Indre (LCI) et Seinemoyenne-Yonne-Loing (SmYL) (voir Fig. 3). Les 240 bassins versants ont été sélectionnés pour répondre à un certain nombre de critères, notamment:

- des influences limitées liées aux activités humaines ou à la neige;

- des surfaces entre 5 et $10000 \mathrm{~km}^{2}$;

- une bonne qualité des données de précipitations, avec des chroniques continues sans lacunes. La densité du réseau pluviométrique définissant la pluie de bassin a également été prise en compte (Ficchì, 2017);

- une bonne disponibilité des données de débit: les stations sélectionnées présentent moins de $35 \%$ de données lacunaires sur chacune des deux périodes test, qui sont respectivement : $01 / 08 / 2005-31 / 7 / 2009$ et $01 / 8 / 2009-31 /$ 7/2013.

Les 26 bassins complémentaires ont davantage été sélectionnés pour leur intérêt en prévision opérationnelle. 
J. Viatgé et al. : La Houille Blanche 2019, 2, 72-80

Tableau 1. Caractéristiques générales de l'échantillon de 266 bassins versants (Source: Pinna, 2017).

\begin{tabular}{llllllll}
\hline & $\begin{array}{l}\text { Superficie } \\
\left(\mathrm{km}^{2}\right)\end{array}$ & $\begin{array}{l}\text { Altitude } \\
(\mathrm{m})\end{array}$ & $\begin{array}{l}\text { Longueur } \\
\text { hydraulique }(\mathrm{km})\end{array}$ & $\begin{array}{l}\text { Densité de } \\
\text { drainage }\left(\mathrm{km}^{-1}\right)\end{array}$ & $\begin{array}{l}\text { Débit moyen } \\
\text { annuel }(\mathrm{mm} / \mathrm{an})\end{array}$ & $\begin{array}{l}\text { Pluie moyenne } \\
\text { annuelle }(\mathrm{mm} / \mathrm{an})\end{array}$ & $\begin{array}{l}\text { ETP moyenne } \\
\text { annuelle }(\mathrm{mm} / \mathrm{an})\end{array}$ \\
\hline Min & 3,5 & 70 & 1,65 & 0,005 & 32 & 627 & 550 \\
Médiane & 373 & 392 & 24,65 & 0,066 & 322 & 916 & 667 \\
Max & 43825 & 1308 & 243,9 & 0,75 & 1480 & 2064 & 859 \\
\hline
\end{tabular}

Au final, l'échantillon sélectionné est représentatif d'une importante variabilité morphologique, hydro-climatique et géographique (voir Tab. 1).

Les données pluviométriques exploitées incluent celles issues de la réanalyse SAFRAN (Vidal et al., 2010) et des données du réseau de pluviographes de Météo-France. Les données hydrométriques sont issues de la banque HYDRO (www.hydro.eaufrance.fr; Leleu et al., 2014). Les données d'ETP ont été calculées sur la base des températures issues de SAFRAN par la formule d'Oudin et al. (2005). Une procédure automatique de sélection d'événement a été mise en œuvre (voir Ficchì et al., 2016). Cela a permis de sélectionner les dix plus forts événements de crue sur chaque bassin.

\section{Méthodologie}

\subsection{Procédure de test}

Le modèle GRP a été testé avec une procédure classique de calages-contrôles croisés (Klemeš, 1986) sur les deux périodes d'évaluation. Chaque fois, le modèle a réalisé de manière rétrospective des prévisions à chaque pas de temps, pour des horizons allant de 1 à $72 \mathrm{~h}$. Le scénario de pluie future considéré ici correspond aux pluies observées a posteriori, ce qui permet de limiter l'incertitude liée à la prévision des pluies.

En calage, les paramètres des modèles sont optimisés en utilisant une erreur quadratique calculée à l'échéance sélectionnée, ici le temps de réaction du bassin versant estimé comme le décalage $\Delta t$ qui maximise la corrélation entre les séries de pluie et de débit (voir Ficchì, 2017). La procédure de calage est une méthode de recherche locale développée à Irstea.

\subsection{Critères d'évaluation}

Les prévisions ont été évaluées à différents horizons : 1, 3, $6,9,12,24$ et $48 \mathrm{~h}$. Leur qualité a été estimée sur la base de critères continus (calculés sur toute la période) et événementiels (sur les dix événements sélectionnés), notamment:

- l'erreur absolue relative moyenne (EARM) à l'échéance choisie: il s'agit de l'erreur absolue moyenne, normalisée par le débit moyen observé ;

- un critère d'erreur relative en amplitude $\Delta Q$ défini comme la différence entre les pics de crue observés et prévus, normalisée par le pic de débit observé.

D'autres critères, non présentés dans le cadre de cet article, ont également été utilisés (critères de persistance, de franchissement de seuil, de retard des pointes de crue, de retard à la montée).

\section{Résultats}

Les travaux de test du modèle GRP pour le rendre plus flexible temporellement, et potentiellement plus performant, ont porté sur de nombreuses versions, détaillées par Pinna (2017). Ici, nous ne présenterons que les options qui ont paru les plus prometteuses. Dans la suite, GRP0 désigne le modèle initial, GRPI désigne le modèle auquel on a ajouté un réservoir d'interception de capacité fixe non nulle, et les compléments du nom de version indiquent les paramètres laissés à leur valeur obtenue en calage en simulation (sans assimilation de données) lors du calage séquentiel.

La figure 4 montre pour diverses échéances les distributions du critère EARM sur les 266 bassins pour différentes versions du modèle. Toutes les versions modifiées comportent quatre paramètres (exceptée la version GR3PI), la capacité du réservoir de production ayant été laissée libre. Les résultats indiquent que (Pinna, 2017):

- l'ajout d'un réservoir d'interception, de capacité fixe (évaluée préalablement de manière climatique), apporte de légères améliorations des performances. Il permet également d'avoir des valeurs de CORR légèrement plus proche de 1 (non montré ici), confirmant l'effet de compensation de l'absence du réservoir par ce paramètre;

- l'optimisation du paramètre gérant la capacité du réservoir de production apporte une amélioration modeste des performances (voir Pinna, 2017, pour des résultats complets);

- des versions traitées par une stratégie de calage séquentiel permettent une amélioration des performances. Le calage du paramètre ROUT sans assimilation conduit à une valeur du paramètre CORR plus proche de 1 en moyenne, diminuant ainsi l'erreur en amplitude. En outre, le calage en simulation du paramètre $\mathrm{TB}$ permet d'améliorer légèrement la restitution temporelle des événements en réduisant le retard à la montée et entre le pic de crue prévu et observé (non montré ici). La version combinant le calage des paramètres ROUT et TB sans assimilation donne les meilleurs gains de performance. L'assimilation masque probablement une partie de l'information dont aurait besoin le modèle pour bien caler ces paramètres dynamiques de la relation pluie-débit.

La figure 5 illustre les résultats de versions modifiées de GRP intégrant des fonctions d'échange en eau agissant sur la pluie efficace $(P r)$ à la place de la simple correction par CORR: l'une (FE) correspondant à la fonction actuelle du modèle GR4J, l'autre (FL) étant une version linéaire de la précédente, comme suggéré par Le Moine (2008), chacune 


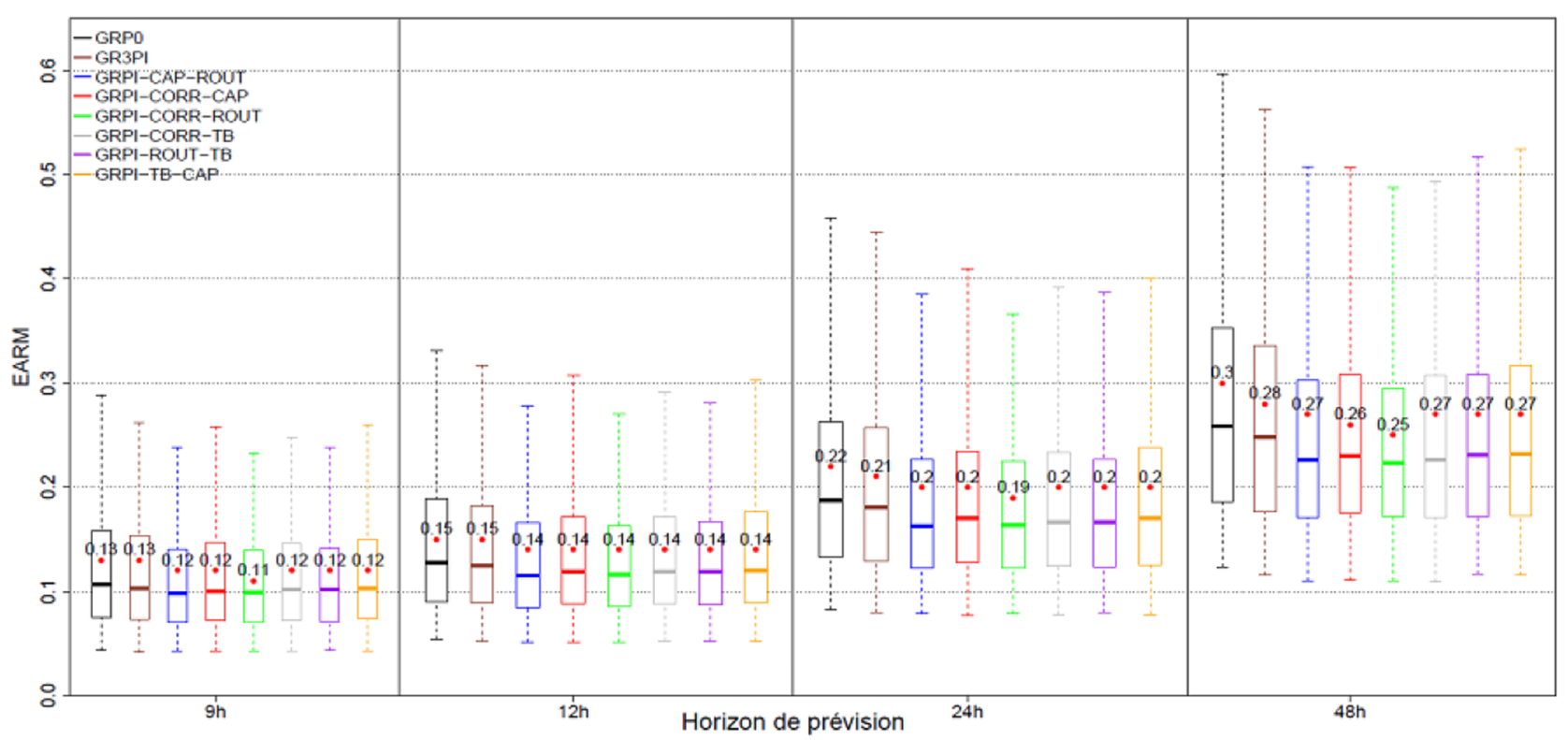

Fig. 4. Distributions du critère continu EARM. Critère obtenu en phase de contrôle à quatre échéances $(9,12,24$ et 48 h) sur les 266 bassins pour différentes versions du modèle GRP (la valeur optimale est 0) (Source : Pinna, 2017).

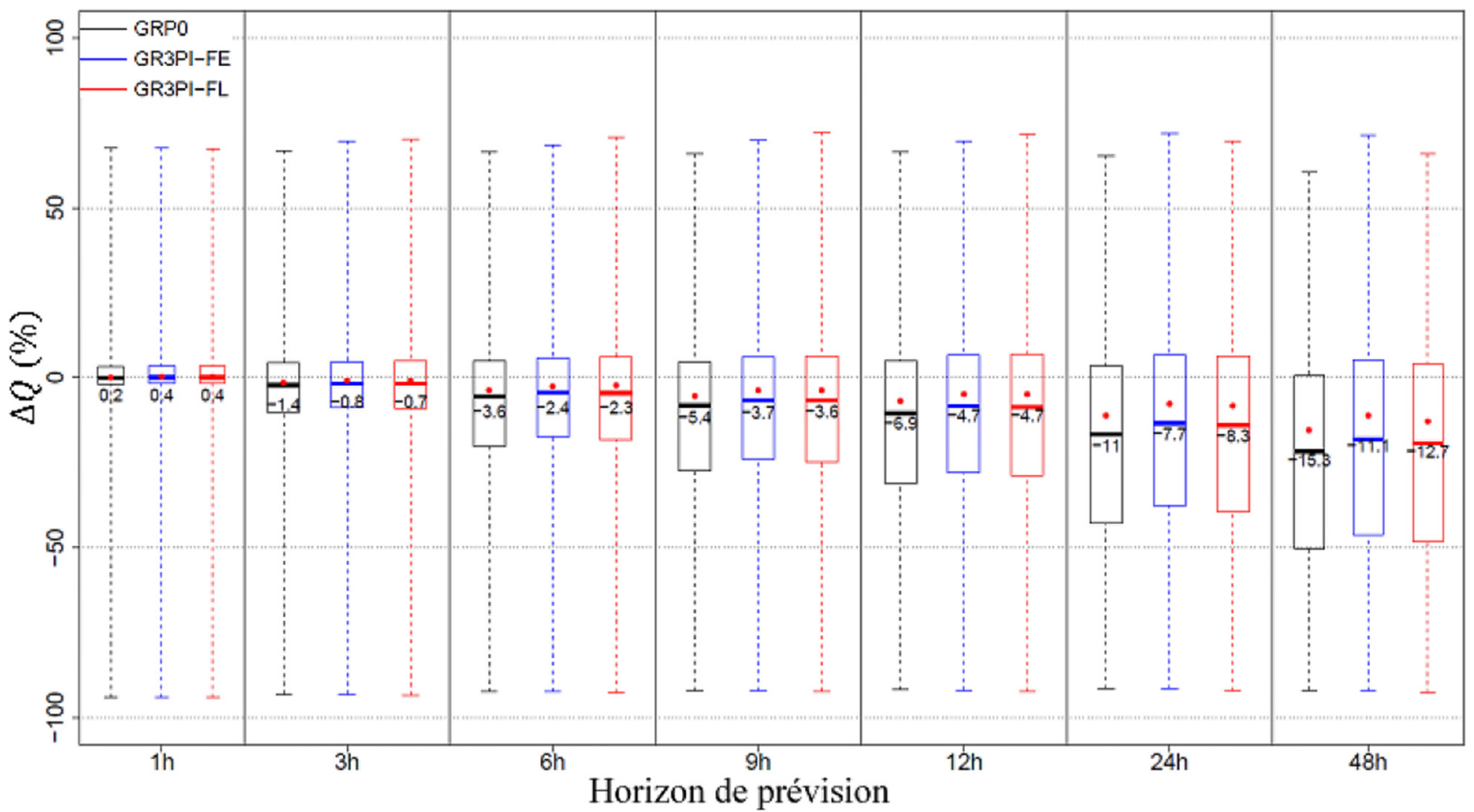

Fig. 5. Distributions du critère événementiel $\Delta Q$. Critère obtenu en phase de contrôle à différentes échéances $(1,3,6,9,12,24$ et 48 h) sur les 266 bassins pour deux versions du modèle GRP où le paramètre CORR est remplacé par une fonction d'échange (la valeur optimale est 0 ) (Source : Pinna, 2017).

n'ayant qu'un seul paramètre libre. Les résultats montrent que cette modification de la fonction d'échange permet une diminution de la sous-estimation des volumes de crue. Un effet induit de cette modification est une réduction du retard à la montée (voir Pinna, 2017).

\section{Conclusions et perspectives}

Les résultats présentés ici suggèrent qu'une version intégrant un réservoir d'interception de capacité fixe (estimée climatiquement) et une modification de la formulation de 
l'ajustement des pluies efficaces permet des gains de performance significatifs, notamment en crue, en limitant la tendance à la sous-estimation communément constatée par les SPC dans l'utilisation de GRP, sans introduire de complexité paramétrique supplémentaire (la capacité du réservoir de production restant fixe). L'application d'un calage séquentiel où les paramètres de routage (ROUT et TB) seraient calés en mode simulation, semble également apporter davantage de robustesse dans l'estimation des paramètres.

Les résultats ont montré un gain de performance, plus significatif pour les horizons lointains. Par ailleurs, les résultats obtenus pour les différentes versions montrent que les critères continus sont relativement peu sensibles aux modifications effectuées, contrairement aux critères événementiels.

Les améliorations des performances obtenues sont certes limitées, mais elles n'engendrent pas d'augmentation de la complexité du modèle. On peut donc considérer qu'elles sont obtenues à " moindre coût». De plus, les tests en simulation effectués à plusieurs pas de temps (sur le modèle GR4), non détaillés ici par souci de concision, ont montré que les bénéfices ne sont pas seulement en termes de performance du modèle, mais aussi en termes de stabilité temporelle des flux et paramètres (Ficchì, 2017). Il n'a cependant pas été possible de trouver des relations claires entre les améliorations obtenues et les caractéristiques des bassins versants. L'analyse conjointe des résolutions spatiales et temporelles semble davantage prometteuse pour identifier des liens avec les caractéristiques des bassins, d'après les travaux de Goullet (2016).

Les tests présentés ici ont été réalisés seulement avec des données au pas de temps horaire. Ils doivent être étendus à d'autres pas de temps infra ou supra-horaire, comme cela avait été fait dans les travaux de Ficchì (2017), de manière à confirmer leur transposabilité temporelle, mais les modifications doivent d'ores et déjà pouvoir améliorer la version opérationnelle de GRP. Des tests sont prévus très prochainement à ce sujet. Le développement d'une version opérationnelle multi-pas de temps du modèle est d'ailleurs en cours, incluant la possibilité de travailler sur des pas de temps allant de l'infra-horaire au journalier. À plus long terme, l'ensemble de ces modifications pourrait être intégrée à la version semidistribuée du modèle. Actuellement, le modèle TGR, version semi-distribuée opérationnelle, utilise une structure modifiée du modèle GRP. L'introduction de ces modifications pourrait améliorer similairement ses performances.

Remerciements. Les auteurs remercient le SCHAPI pour son soutien financier au développement du modèle GRP et aux travaux de Master du deuxième auteur et de thèse du troisième auteur. Les données hydrologiques et climatiques nécessaires au test du modèle ont été fournies respectivement par la banque HYDRO gérée par le SCHAPI, et par Météo-France. Les auteurs remercient également les SPC qui ont contribué, par leurs retours et leurs suggestions, à faire avancer la réflexion sur le modèle GRP. Enfin, les auteurs remercient le relecteur anonyme de l'article dont les suggestions ont permis de clarifier le texte.

\section{Références}

Berthet L. 2010. Prévision des crues au pas de temps horaire : pour une meilleure assimilation de l'information de débit dans un modèle hydrologique. Thèse de Doctorat, Cemagref (Antony), AgroParisTech (Paris), Paris, 603 p. Disponible sur https:// webgr.irstea.fr/wp-content/uploads/2012/07/2010-BERTHETTHESE.pdf.

Ficchì A. 2017. An adaptive hydrological model for multiple timesteps: Diagnostics and improvements based on fluxes consistency. Thèse de doctorat, Irstea (Antony), GRNE (Paris), $281 \mathrm{p}$. Disponible sur https://webgr.irstea.fr/wp-content/uploads/2017/ 09/2017-Thesis_final_Andrea_Ficchi_Irstea_2017.pdf.

Ficchì A, Perrin C, Andréassian V. 2016. Impact of temporal resolution of inputs on hydrological model performance: An analysis based on 2400 flood events. J Hydrol 538: 454-470. DOI: 10.1016/j.jhydrol.2016.04.016.

Ficchì A, Perrin C, Andréassian V. 2019. Hydrological modelling at multiple sub-daily time steps: Model improvement via flux-matching. J Hydrol 575: 1308-1327. DOI: 10.1016/j.jhydrol.2019.05.084.

Furusho C, Perrin C, Lamblin R, Dorchies D, Berthet L, Andréassian V. 2015. Dynamique de collaboration entre acteurs opérationnels et scientifiques pour une amélioration des systèmes de prévision des crues en France, (en ligne), Rev SET, 6p. Disponible sur http://www.set-revue.fr/dynamique-de-collabora tion-entre-acteurs-operationnels-et-scientifiques-pour-une-ame lioration-des (dernière consult. 2015/6/11).

Furusho C, Perrin C, Viatgé J, Lamblin R, Andréassian V. 2016. Synergies entre acteurs opérationnels et scientifiques au service de l'amélioration de la prévision des crues. La Houille Blanche 4: 510. DOI: $10.1051 / \mathrm{lhb} / 2016033$.

Furusho C et al. 2017. Calage et application opérationnelle du modèle de prévision de crue GRP-Manuel d'utilisation. Version 2016. r1460, Irstea, Antony, France, $81 \mathrm{p}$.

Goullet J. 2016. À la recherche des résolutions spatiales et temporelles caractéristiques du comportement hydrologique d'un bassin versant. Mémoire de Master 2 SDUEE, UPMC, Irstea, Antony, 53 p. Disponible sur https://webgr.irstea.fr/wp-content/ uploads/2017/06/an2016-pub00053045.pdf.

Klemeš V. 1986. Operational testing of hydrological simulation models. Hydrol Sci J 31(1): 13-24.

Le Moine N. 2008. Le bassin versant de surface vu par le souterrain: une voie d'amélioration des performances et du réalisme des modèles pluie-débit? Thèse de Doctorat, Université Pierre et Marie Curie (Paris), Cemagref (Antony), 324 p. Disponible sur https:// webgr.irstea.fr/wp-content/uploads/2012/07/2008-LE_MOINETHESE.pdf.

Leleu I, Tonnelier I, Puechberty R et al. 2014. Re-founding the national information system designed to manage and give access to hydrometric data. La Houille Blanche, 25-32. DOI: 10.1051/lhb/ 2014004.

Mathevet T. 2005. Quels modèles pluie-débit globaux pour le pas de temps horaire? Développement empirique et comparaison de modèles sur un large échantillon de bassins versants. Thèse de Doctorat, ENGREF (Paris), Cemagref (Antony), France, 463 p. Disponible sur https://webgr.irstea.fr/wp-content/uploads/2012/07/ 2005-MATHEVET-THESE.pdf.

Mouelhi S. 2003. Vers une chaîne cohérente de modèles pluie-débit conceptuels globaux aux pas de temps pluriannuel, annuel, mensuel et journalier. Thèse de Doctorat, ENGREF, Cemagref, Antony, France, 323 p. Disponible sur https://webgr.irstea.fr/wp-content/ uploads/2012/07/2003-MOUELHI-THESE.pdf.

Obled C, Zin I, Hingray B. 2009. Choix des pas de temps et d'espace pour des modélisations parcimonieuses en hydrologie des crues. La Houille Blanche 5: 81-87. DOI: 10.1051/lhb/ 2009059.

Oudin L, Hervieu F, Michel C et al. 2005. Which potential evapotranspiration input for a rainfall-runoff model? Part 
2-Towards a simple and efficient PE model for rainfall-runoff modelling. J Hydrol 303(1-4): 290-306. DOI: 10.1016/j.jhy drol.2004.08.026.

Perrin C, Michel C, Andréassian V. 2003. Improvement of a parsimonious model for streamflow simulation. J Hydrol 279: 275289. DOI: $10.1016 / \mathrm{S} 0022-1694(03) 00225-7$.

Pinna T. 2017. Peut-on améliorer les performances du modèle GRP pour la prévision des crues? Tests sur un échantillon de bassins versants français. Mémoire de Master 2 SDUEE, UPMC, Irstea, Antony, 53 p. Disponible sur https://webgr.irstea.fr/wp-content/ uploads/2018/07/Memoire_M2_Pinna_VF.pdf.

Santos L, Thirel G, Perrin C. 2018. State-space representation of a bucket-type rainfall-runoff model: A case study with State-Space GR4 (version 1.0). Geosci Model Dev 11: 1591-1605. DOI: 10.5194/gmd-11-1591-2018.

Tangara M. 2005. Nouvelle méthode de prévision de crue utilisant un modèle pluie-débit global. Thèse de Doctorat, Cemagref, Antony, EPHE, Paris, $374 \mathrm{p}$.

Vidal JP, Martin E, Franchistéguy L, Baillon M, Soubeyroux JM. 2010. A 50-year high-resolution atmospheric reanalysis over France with the Safran system. Int J Climatol 30(11): 1627-1644. DOI: $10.1002 /$ joc.2003.

\section{Annexe 1 Description du modèle GRP multi- pas de temps}

Cette annexe présente le fonctionnement détaillé du modèle GRP, explicitant la dépendance de la formulation au pas de temps, en s'inspirant des travaux de Ficchì (2017, Annexe G). Nous présentons ici une version intégrant un réservoir d'interception de capacité non nulle, telle que suggérée par Ficchì (2017). Notons qu'une écriture en représentation d'états permettrait également d'avoir un traitement plus rigoureux de cette question du pas de temps (voir Santos et al., 2018).

\section{Interception}

À chaque pas de temps, les précipitations $P$ entrent dans le réservoir d'interception. L'évapotranspiration $E$ agit sur son contenu à un taux potentiel. La capacité de ce réservoir (en $\mathrm{mm})$ est déterminée climatiquement de manière à retrouver les quantités d'eau moyennes interceptées au pas de temps journalier (avec un réservoir de capacité nulle). Cette capacité dépendra donc du pas de temps choisi. À chaque pas de temps, le réservoir produit soit un surplus de pluie $P n$ (débordement au-dessus de la capacité), soit un reliquat éventuel d'ETP $(E n)$.

\section{Production}

La pluie issue du réservoir d'interception $(P n)$ est alors divisée en deux parties en fonction du taux de remplissage $S /$ CAP du réservoir de production, $S(\mathrm{~mm})$ étant le niveau du réservoir et $\mathrm{CAP}(\mathrm{mm})$ sa capacité qui est indépendante du pas de temps (fixée à $275 \mathrm{~mm}$ ). Une partie $(P S$, Eq. (1)) alimente ce réservoir et l'autre $(P n-P S)$ alimente l'écoulement. Le reliquat d'ETP, En, contribue à la vidange du réservoir de production par évapotranspiration réelle $(E s$, Eq. (2)).

$$
\begin{gathered}
P S=\frac{\mathrm{CAP} \cdot\left(1-\left(\frac{S}{\mathrm{CAP}}\right)^{2} \cdot \tanh \left(\frac{P n}{\mathrm{CAP}}\right)\right)}{1+\frac{S}{\mathrm{CAP}} \cdot \tanh \left(\frac{P n}{\mathrm{CAP}}\right)} \text { et } E s=0, \\
E s=\frac{S \cdot\left(2-\frac{S}{\mathrm{CAP}}\right) \cdot \tanh \left(\frac{E n}{\mathrm{CAP}}\right)}{1+\left(1-\frac{S}{\mathrm{CAP}}\right) \cdot \tanh \left(\frac{E n}{\mathrm{CAP}}\right)} \text { et } P s=0 .
\end{gathered}
$$

Le niveau est actualisé par:

$$
S=S-E s+P s
$$

Le réservoir se vidange ensuite par une percolation Perc:

$$
\text { Perc }=S \cdot\left\{1-\left[1+\left(\frac{S}{\beta \cdot \mathrm{CAP}}\right)^{4}\right]^{-\frac{1}{4}}\right\},
$$

avec $\beta$ est une constante fonction du pas de temps. Le niveau est de nouveau actualisé par:

$$
S=S-\text { Perc. }
$$

La quantité d'eau totale allant alimenter l'écoulement $(P r$, Eq. (6)) est multipliée par le facteur d'ajustement, CORR (-):

$$
\begin{gathered}
P r=\text { Perc }+(P n-P s), \\
P r^{\prime}=P r \cdot \text { CORR. }
\end{gathered}
$$

\section{Transfert}

\section{Hydrogramme unitaire}

L'hydrogramme unitaire (HU) permet de répartir la pluie efficace $P r^{\prime}$ de manière discrète. Le calcul des ordonnées de l'hydrogramme unitaire est réalisé à partir de la fonction cumulée (courbe en $S$ ) définie par:

$$
\mathrm{SH}(k)= \begin{cases}0 & \text { si } k \leq 0 \\ \frac{k^{1.25}}{k^{1.25}+(\mathrm{TB}-k)^{1.25}} & \text { si } 0 \leq k \leq \mathrm{TB} \\ \text { si } \mathrm{TB} \leq k,\end{cases}
$$

où TB (ayant la dimension du pas de temps considéré) est le temps de base de l'hydrogramme calé par le modèle. Les ordonnées de l'hydrogramme unitaire discret sont calculées par différences successives de la courbe en $S$ aux temps entiers :

$$
\mathrm{HU}(k)=\mathrm{SH}(k)-\mathrm{SH}(k-1) .
$$

Le débit transféré par l'HU est obtenu par convolution des pluies efficaces antérieures:

$$
\mathrm{qhu}_{t}=\sum_{k=1}^{m} \operatorname{Pr}_{t-k+1}^{\prime} \cdot \mathrm{HU}(k),
$$

où $m$ correspond au nombre d'ordonnées non nulles de l'hydrogramme. 


\section{Réservoir de routage}

Le niveau $R$ du réservoir de routage est régi par deux équations :

$$
\left\{\begin{array}{l}
Q(t)=\alpha * R(t)^{2} \\
d R=-Q(t) d t
\end{array} .\right.
$$

L'intégration sur le pas de temps permet d'obtenir la valeur du niveau du réservoir en fin de pas de temps $R_{j+1}$ en fonction de celui en début de pas de temps $R_{j}$ (Eq. (13)) en posant ROUT $=\frac{1}{\alpha \Delta}$, que l'on définit comme la capacité du réservoir de routage à un pas de temps :

$$
R_{j+1}=\frac{R_{j} \cdot \mathrm{ROUT}}{\mathrm{ROUT}+R_{j}}
$$

Le débit $Q$ en sortie est donc donné par:

$$
Q=R_{j}-R_{j+1}=\frac{R_{j}^{2}}{R_{j}+\mathrm{ROUT}} .
$$

\section{Assimilation de données}

L'exploitation du dernier débit observé se fait par une procédure d'assimilation (ou de mise à jour), qui se déroule en deux temps au sein de GRP.

\section{Mise à jour du réservoir de routage}

La mise à jour directe du réservoir de routage permet de recalculer son niveau de manière à générer un débit exactement égal au dernier débit observé à l'instant de prévision. Le niveau du réservoir $R^{*}{ }_{t}$ mis à jour est donné par:

$$
\begin{aligned}
R_{* t} & =R_{*_{t-1}}-Q_{\mathrm{obst}} \\
& =\frac{\sqrt{Q_{\mathrm{obst}}^{2}}+4 \cdot \operatorname{ROUT} \cdot Q_{\mathrm{obst}}-Q_{\mathrm{obst}}}{2} .
\end{aligned}
$$

Il sert de condition initiale au début du premier pas de temps de prévision.

\section{Correction des sorties}

Les débits prévus sont corrigés par un procédé simple de type autorégressif, qui vise à exploiter la connaissance de l'erreur antérieure du modèle (Tangara, 2005):

$$
\hat{Q}_{P_{t \rightarrow t+L}}=Q_{P_{t \rightarrow t+L}} \times\left(\frac{Q_{\mathrm{O}_{t}}}{Q_{P_{t-1}}}\right)^{\alpha},
$$

où $\hat{Q}_{P_{t \rightarrow t+L}}$ est le débit prévu au pas de temps $t$ pour le pas de temps $t+L$ ( $L$ : délai de prévision), $Q_{\mathrm{O} t}$ est le débit observé à l'instant $t, Q_{P_{t-1 \rightarrow t}}$ est le débit prévu avant correction, et $\alpha$ est un coefficient fixé à 0,45 . Une correction par réseaux de neurones artificiels est aussi possible (Berthet, 2010).

Citation de l'article : Viatgé J, Pinna T, Ficchì A, Perrin C, Dorchies D, Garandeau L, Tilmant F. 2019. Vers une plus grande flexibilité temporelle du modèle opérationnel de prévision des crues GRP. La Houille Blanche 2: 72-80. 